\title{
Psychomotor Vigilance Impairment During Total Sleep Deprivation Is Exacerbated in Sleep-Onset Insomnia
}

This article was published in the following Dove Press journal:

Nature and Science of Sleep

\author{
Devon A Hansen (D) ${ }^{1,2}$ \\ Matthew E Layton ${ }^{1-3}$ \\ Samantha M Riedy ${ }^{1,2}$ \\ Hans PA Van Dongen (iD) ${ }^{1,2}$ \\ 'Sleep and Performance Research Center, \\ Washington State University, Spokane, \\ WA, USA; ${ }^{2}$ Department of Medical \\ Education and Clinical Sciences, Elson \\ S. Floyd College of Medicine, Washington \\ State University, Spokane, WA, USA; \\ ${ }^{3}$ Program of Excellence in Addictions \\ Research, College of Nursing, \\ Washington State University, Spokane, \\ WA, USA
}

\begin{abstract}
Purpose: Individuals with primary insomnia frequently report cognitive impairment as a next-day consequence of disrupted sleep. Studies attempting to quantify daytime impairment objectively in individuals with insomnia have yielded mixed results, with evidence suggesting impairments in aspects of executive functioning but not psychomotor vigilance. It has been suggested that persons with insomnia may have latent performance deficits for which they would be able to compensate effectively under normal daytime circumstances suggesting that any such deficits may be exposed through perturbation. In this context, we used a laboratory-based total sleep deprivation (TSD) paradigm to investigate psychomotor vigilance performance in individuals with chronic sleep-onset insomnia as compared to healthy normal controls.
\end{abstract}

Participants and methods: Fourteen participants, seven individuals with chronic sleeponset insomnia (ages 24-40y) and seven age-matched, healthy normal sleepers completed a highly controlled in-laboratory study involving $38 \mathrm{~h}$ of TSD. A $10 \mathrm{~min}$ and a $3 \mathrm{~min}$ version of the psychomotor vigilance test (PVT) were administered every $3 \mathrm{~h}$ during TSD.

Results: In both the individuals with sleep-onset insomnia and the age-matched normal sleepers, lapses of attention and false starts on the PVT were relatively infrequent during the first $16 \mathrm{~h}$ of the TSD period, but increased significantly when wakefulness was extended beyond $16 \mathrm{~h}$. However, the effects of TSD on PVT performance were considerably exacerbated in the sleep-onset insomnia group, which showed about twice as many lapses of attention, more than twice as many false starts, and approximately twice as big a time-ontask effect on the 10 min PVT as the age-matched normal sleepers group, with similar findings on the 3 min PVT.

Conclusion: These findings indicate that daytime impairment reported by individuals with sleep-onset insomnia has an objective performance component that is exposed during TSD. Thus, persons with sleep-onset insomnia could be at increased risk of performance impairment in settings that involve extended wakefulness. This underscores the importance of treating insomnia and suggests that laboratory sleep deprivation studies could serve to document the effectiveness of treatment approaches.

Keywords: bedtime-specific hyperarousal, chronic insomnia, cognitive performance, extended wakefulness, primary insomnia, vulnerability to sleep loss

\section{Introduction}

Chronic insomnia is a serious public health concern affecting up to $25 \%$ of the US population, with an estimated societal cost of over 60 billion dollars annually. ${ }^{1}$ Chronic insomnia is defined as difficulty initiating or maintaining sleep at least 3
Correspondence: Devon A Hansen Washington State University Spokane,

412 E. Spokane Falls Blvd., Spokane, WA 99202, USA

Tel + I-509-358-7754

$\mathrm{Fax}+\mathrm{I}-509-358-7810$

Email devon.hansen@wsu.edu 
days per week for at least 3 months despite adequate opportunity and sleep environment, with reported nextday consequences causing significant impairment in social, emotional, occupational or cognitive functioning. ${ }^{2}$

Individuals with chronic insomnia frequently report daytime cognitive impairment, but this has proven to be difficult to measure objectively. The literature on this issue is mixed, with most of the evidence suggesting no significant impairment in psychomotor vigilance ${ }^{3-6}$ but some evidence suggesting deficits in (typically poorly defined) higher-level neurobehavioral functions. $^{5-9}$ Daytime impairment in psychomotor vigilance has been observed in patients with insomnia undergoing sleep restriction therapy, ${ }^{10}$ but it is not clear whether this impairment is any different from what has been observed in healthy normals subjected to sleep restriction. ${ }^{11,12}$

The lack of clarity regarding the cognitive consequences of chronic insomnia may stem, in part, from failure to differentiate stable insomnia subtypes - sleep-onset insomnia and sleepmaintenance insomnia. ${ }^{13}$ In particular, individuals with sleeponset insomnia have been reported to be able to compensate effectively for psychomotor vigilance performance impairment during the normal day, ${ }^{14}$ even though they may in fact have latent performance deficits. This possibility could be investigated by exposing any such latent deficits by means of an experimental perturbation. To this end, we compared psychomotor vigilance performance impairment in individuals with chronic sleep-onset insomnia to age-matched healthy normal controls in a laboratory-based total sleep deprivation (TSD) paradigm.

\section{Materials and Methods}

\section{Participants}

A group of 7 individuals with primary sleep-onset insomnia (6 female, 1 male), average age 29.0 y (SD, 6.2 y), completed the highly controlled, in-laboratory study. A separate group of 7 age-matched, healthy normal sleepers (4 female, 3 male), average age 29.0 y (SD, $6.6 \mathrm{y}$ ), was drawn from a larger group of 16 who also completed the study ${ }^{15}$ and served as a control group. Age-matching was precise to within a year (SD of difference, $0.8 \mathrm{y}$ ). Participants were financially compensated for their study participation. They gave written, informed consent, and the study was approved by the Institutional Review Board (IRB) of Washington State University. All experimental procedures were in accordance with the Declaration of Helsinki.

Participants underwent a rigorous screening process, which included a telephone interview and two in-office screening sessions to ensure they met study eligibility criteria. They were in the age range of 22-40 y. They were physically and psychologically healthy, and female participants were not pregnant. Besides chronic insomnia (for the sleep-onset insomnia group only), participants had no sleep or circadian disorders, as confirmed with validated questionnaires, clinical interview by the physician of record for the study, and laboratory baseline polysomnography. Participants were free of drugs and alcohol, as assessed by physical examination, blood and urine chemistry, and breathalyzer test. They had no clinically relevant history of psychiatric illness and no clinically relevant history of brain injury. They had no history of drug or alcohol abuse in the past year and no history of methamphetamine abuse. They were not current tobacco users and reported no current medical or drug treatment (excluding oral contraceptives). They were not vision impaired or their vision was corrected to normal. Participants had not traveled across time zones within 1 month of entering the study and reported no shift work within 3 months of entering the study. Their habitual wake time was between 06:00 and 09:00. The normal sleeper group reported habitual sleep durations between 6 and $10 \mathrm{~h}$ per day; this inclusion criterion was not used for the sleep-onset insomnia group.

Participants with sleep-onset insomnia were additionally screened to meet the criteria of Chronic Insomnia Disorder described in the International Classification of Sleep Disorders 3 (ICSD-3), ${ }^{2}$ with a specific primary complaint of trouble initiating sleep occurring at least three times per week for a period of at least 3 months. Participants were considered to have sleep-onset insomnia if they had an average self-reported nighttime sleep latency of at least $30 \mathrm{~min}$. To ensure that sleep disturbance was not due to delayed sleep phase, participants in the sleep-onset insomnia group were also required to have an average habitual bedtime between 21:00 and 23:00 and not go to bed any later than 01:00.

Participants were instructed to keep their habitual sleep schedule (within \pm 30 mins) and to abstain from caffeine, alcohol, tobacco, drug use, and napping during the 7 days prior to admission into the laboratory. During these 7 days, they completed a sleep/wake diary and called a time-stamped voicemail box to report their bedtimes upon awakening in the morning and before going to bed in the evening. They also wore a wrist actigraph to measure their rest/activity patterns.

A number of questionnaires were administered prior to the laboratory study in order to characterize sleep-related traits. The Epworth Sleepiness Scale (ESS) ${ }^{16}$ is an 8 -item scale assessing excessive daytime sleepiness. The Pittsburg Sleep Quality Index (PSQI) ${ }^{17}$ is a 19-item instrument measuring 
Table I Participant Characteristics

\begin{tabular}{|l|l|l|}
\hline & $\begin{array}{l}\text { Sleep-Onset } \\
\text { Insomnia } \\
\text { Group }\end{array}$ & $\begin{array}{l}\text { Healthy } \\
\text { Control } \\
\text { Group }\end{array}$ \\
\hline N & 7 & 7 \\
Age (y) & $29.0 \pm 6.2$ & $29.0 \pm 6.6$ \\
Gender (M, F) & 1,6 & 3.4 \\
Body Mass Index (kg/m ${ }^{2}$ ) & $26.3 \pm 6.1$ & $25.1 \pm 2.1$ \\
Habitual caffeine use (drinks per & $0.8 \pm 0.6$ & $0.9 \pm 0.8$ \\
day) & $8.0 \pm 4.6 *$ & $3.0 \pm 1.0$ \\
Epworth Sleepiness Scale & $37.0 \pm 6.6$ & $39.4 \pm 5.3$ \\
Composite Scale of & & \\
Morningness & $10.4 \pm 3.1 * * *$ & $2.0 \pm 1.0$ \\
Pittsburg Sleep Quality Index & $28.0 \pm 9.1$ & - \\
Insomnia Severity Index & & \\
\hline Sleep history (sleep diary) & $9.2 \pm 2.1$ & $8.3 \pm 0.8$ \\
Time in bed (h) & $6.5 \pm 0.8$ & $7.3 \pm 0.7$ \\
Sleep duration (h) & $72.5 \pm 13.1 * * *$ & $88.0 \pm 2.7$ \\
Sleep efficiency (\%) & $1.8 \pm 1.6 *$ & $0.2 \pm 0.1$ \\
Sleep latency (h) & $0.9 \pm 0.3$ & $0.8 \pm 0.3$ \\
Intermittent wakefulness (h) & & \\
\hline Sleep history (actigraphy) & $7.0 \pm 0.8$ & $7.0 \pm 0.8$ \\
Sleep duration (h) & $22: 09 \pm 0.6 * *$ & $22: 53 \pm 1.3$ \\
Bedtime (h) & $07: 07 \pm 1.9$ & $07: 19 \pm 0.8$ \\
Waketime (h) & $0.6 \pm 0.3$ & $0.7 \pm 0.4$ \\
\hline Baseline in-laboratory sleep (PSG) & & 10.0 \\
Time in bed (h) & $9.1 \pm 0.4$ & $9.1 \pm 0.5$ \\
Total sleep time (h) & $91.1 \pm 3.5$ & $91.0 \pm 5.1$ \\
Sleep efficiency (\%) & Sleep latency (h) \\
WASO (h) & $0.3 \pm 0.3$ & $0.3 \pm 0.2$ \\
\hline
\end{tabular}

Notes: Means $( \pm \mathrm{SD})$ are shown. Group differences were assessed by independent samples t-test.

Abbreviations: $* \mathrm{P}<0.05$, $* * \mathrm{P}<0.01$, $* * * \mathrm{P}<0.001$; PSG, polysomnography; WASO, wakefulness after sleep onset.

sleep quality in the previous month. The Composite Scale of Morningness $(\mathrm{CSM})^{18}$ is a 13 -item scale assessing circadian phase preference. The Insomnia Severity Index (ISI), ${ }^{19}$ which was administered to the sleep-onset insomnia group only, is a 5-item instrument evaluating insomnia severity. Table 1 shows a range of for participant characteristics in the sleeponset insomnia and age-matched healthy control group.

\section{Procedure}

The study took place inside the sleep laboratory of the Sleep and Performance Research Center at Washington State University in Spokane, WA, USA. Participants were studied in groups of up to 4 and were assigned their own room for performance testing and scheduled sleep periods. They were in the laboratory for 5 days (4 nights) for a total of $96 \mathrm{~h}$ consecutively. The laboratory environment was carefully controlled, with fixed ambient temperature $\left(22 \pm 1{ }^{\circ} \mathrm{C}\right)$, and fixed light levels $(<100$ lux $)$ during periods of scheduled wakefulness (lights were off during scheduled sleep periods). Participants were not allowed to use phones, computers, or otherwise communicate with the outside world.

Figure 1 shows a schematic of the laboratory study protocol. On the first day of the study, participants reported to the laboratory at 14:00. To account for the well-documented first-night effect in both healthy and sleep-disordered populations in a laboratory setting, ${ }^{20}$ the first day and night served as a practice and adaptation period. Participants went to bed at 22:00, with scheduled awakening at 08:00 the following day. The second day in the laboratory served as baseline. Participants again went to bed at 22:00 for baseline sleep, with scheduled awakening at 08:00. Beginning on the third day at 08:00, participants underwent $38 \mathrm{~h}$ of TSD. On the fourth day, they went to bed at 22:00 for recovery sleep, with scheduled awakening at 08:00. Participants were discharged from the laboratory on the fifth day at 14:00.

For participants with sleep-onset insomnia, admission into the study on the first day included a review of their sleep diary to verify an average self-reported sleep latency of at least $30 \mathrm{~min}(\mathrm{M}, 1.8 \mathrm{~h}$; SD, $1.6 \mathrm{~h}$; range $0.5 \mathrm{~h}$ to $4.5 \mathrm{~h}$ ), and a review of their sleep diary and wrist actigraphy data to verify that habitual bedtime was between 21:00 and 23:00 on

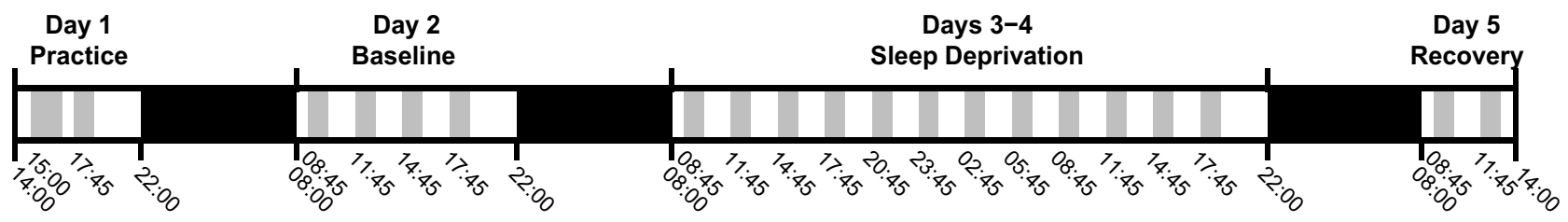

Figure I Schematic of the 5-day (4-night) laboratory study protocol.

Notes: Participants entered the laboratory at $14: 00$ on day I and remained in the laboratory until $14: 00$ on day 5 . Distinct phases of the study are indicated above the schematic. The tick marks below the schematic indicate the study start and end times (14:00) and the bedtimes (22:00) and wake-up times (08:00). All other indicated clock times mark the start times of performance test blocks. Black: scheduled sleep; gray: performance testing. 
average and no later than 01:00 on any day. For the normal sleepers, admission included a review of their sleep diary and wrist actigraphy to verify that bedtimes and wake-up times were consistent (no more than $\pm 30 \mathrm{~min}$ from their selfreported habitual times on average).

In their assigned room, participants were trained on a psychomotor vigilance test (PVT) by a member of the research team. Participants were also trained on a 20 min neuropsychological performance test battery, the data of which are not a focus of this paper and will be reported elsewhere. Following training and a break of at least $45 \mathrm{~min}$, participants practiced each of the performance tasks on their own.

During all scheduled wakefulness from the second day at 08:45 through to the fifth day at 11:45, performance tasks were administered every $3 \mathrm{~h}$ (except in the $3 \mathrm{~h}$ period right before bedtime). See Figure 1. Each test block included a 3 min version of the PVT (PVT-B), ${ }^{21}$ followed by a $10 \mathrm{~min}$ break, a $10 \mathrm{~min}$ version of the $\mathrm{PVT}^{22}$ a 20 min break, and the 20 min neuropsychological test battery. Participants performed all tasks at a desk in their assigned room in the laboratory. They were instructed to give their best effort during performance testing.

All in-laboratory sleep periods were recorded by polysomnography (PSG; Nihon Kohden, Irvine, CA) and scored manually based on AASM criteria. ${ }^{23}$ The PSG montage included frontal (F3, F4), central (C3, C4), and occipital (O1, O2) EEG; bilateral EOG; and submental and masseter EMG. The EEG and EOG electrodes were referenced to the contralateral mastoid. All records were scored by the same registered polysomnographic technologist.

Throughout the laboratory study, meals were served every $4 \mathrm{~h}$ of scheduled wakefulness; caffeine was not permitted. During scheduled wakefulness between performance testing, participants engaged in non-vigorous activities inside the laboratory (e.g., watching movies, playing board games, talking with other participants and/or staff). Participants were behaviorally monitored by trained research assistants throughout the laboratory study. The research assistants ensured that the participants did not fall asleep during scheduled wakefulness, including the $38 \mathrm{~h}$ TSD period. During performance testing, participants were monitored with camera views of their rooms (wide angle) and of their faces (narrow angle).

\section{Measures}

The PVT - a serial reaction time task with high stimulus density $^{22}$ - was used to measure performance impairment resulting from sleep deprivation. Participants were instructed to respond as quickly as possible, while avoiding false starts, to a visual stimulus presented at random inter-trial intervals (ITIs). The PVT was administered on a laptop computer (Pulsar Informatics, Inc., Philadelphia, PA) as a $10 \mathrm{~min}$ version with ITIs ranging from $2 \mathrm{~s}$ to $10 \mathrm{~s}$, and also on a touch-screen smartphone (Pulsar Informatics, Inc., Philadelphia, PA) as a 3 min version with ITIs ranging from $2 \mathrm{~s}$ to $5 \mathrm{~s}$. Participants responded to the stimulus by pressing the space bar (on the laptop) or finger-tapping the screen (on the smartphone) using the index finger of their dominant hand.

The 10 min version of the PVT has been shown to be highly sensitive to sleep loss-induced impairment. ${ }^{24}$ It exhibits a time-on-task effect that is amplified by sleep loss. ${ }^{25}$ The 3 min version of the PVT (PVT-B) is less sensitive to sleep loss due to its shorter task duration and consequently restricted time-on-task effect, but the loss of sensitivity is partially mitigated by the shorter ITIs. ${ }^{21}$ Both the 10 min laptop version and the 3 min smartphone version of the PVT were validated in our laboratory in a sample of healthy adults. ${ }^{15}$

\section{Statistical Analyses}

Statistical analyses focused on the $10 \mathrm{~min}$ and $3 \mathrm{~min}$ PVTs that took place during the twelve $3 \mathrm{~h}$ test blocks of the 38 $\mathrm{h}$ TSD period and the two $3 \mathrm{~h}$ test blocks after recovery sleep (see Figure 1). The primary outcome variable was the number of lapses of attention, which was defined as the number of response times $(\mathrm{RTs}) \geq 500 \mathrm{~ms}$ (for the $10 \mathrm{~min}$ PVT) ${ }^{26}$ or $\geq 355 \mathrm{~ms}$ (for the $3 \mathrm{~min}$ PVT). ${ }^{21}$ Other outcome variables were the mean RT, and the number of false starts (responses prior to or within $100 \mathrm{~ms}$ of stimulus presentation). Due to a technical problem, the 10 min PVT in the first test block after recovery sleep was missing for one participant.

To account for both within- and between-subjects variance, PVT outcome measures were analyzed using mixedeffects analysis of variance (ANOVA) ${ }^{27}$ with fixed effects for groups, test blocks, and their interaction, and a random effect over matched participant pairs on the intercept. Because the two groups were age-matched but not sex-matched, analyses were repeated with sex as an additional fixed effect, alone and in two- and three-way interactions with group and test block.

The time-on-task effect was analyzed by dividing the task duration of each version of the PVT into 1 min bins and subjecting the raw RT data to mixed-effects ANOVA, with fixed effects for groups, test blocks, 1 min bins, and their interactions, and a random effect over matched participant pairs on the intercept. 


\section{Results}

Table 2 and Figure 2 show the main results of the study. For number of lapses of attention on the $10 \mathrm{~min}$ PVT across the $38 \mathrm{~h}$ TSD period and following recovery sleep, there was a significant effect of group $\left(\mathrm{F}_{1161}=\right.$ 22.42, $\mathrm{P}<0.001)$ and a significant effect of test block $\left(\mathrm{F}_{13,161}=11.95, \mathrm{P}<0.001\right)$. These effects persisted when sex was included in the analysis. Likewise, for number of lapses of attention on the 3 min PVT across sleep deprivation and following recovery sleep, there was a significant effect of group $\left(\mathrm{F}_{1162}=22.45, \mathrm{P}<0.001\right)$ and a significant effect of test block $\left(\mathrm{F}_{13,162}=5.06, \mathrm{P}<0.001\right)$. When sex was included in the analysis, the effect of group was reduced to a trend $\left(\mathrm{F}_{1134}=2.78, \mathrm{P}=0.098\right)$, while the effect of test block persisted. There was a significant effect of sex on the 3 min PVT $\left(F_{1134}=7.38, P=0.008\right)$, with females exhibiting 2.9 (SE, 1.1) more lapses than males.

In both groups and for both versions of the PVT, the number of lapses was low during the first $16 \mathrm{~h}$ of wakefulness and then increased considerably across the 38 $\mathrm{h}$ TSD period, with peak impairment during the early to late morning and a partial rebound during the subsequent afternoon. Following recovery sleep, the number of lapses returned to the level observed during the first 16 $\mathrm{h}$ of the TSD period, indicating that the performance impairment seen toward the end of the TSD period was specific to sleep loss. Overall, there were fewer lapses of attention on the 3 min PVT than on the 10 min PVT due to the difference in task duration. However, for both versions of the PVT, the sleep-onset insomnia group experienced greater performance impairment during sleep deprivation as compared to the healthy normal sleepers (Figure 2, top).
This pattern of results repeated itself for mean RT (Table 2; Figure 2, middle). On the 10 min PVT, there was a significant effect of group $\left(\mathrm{F}_{1161}=13.66, \mathrm{P}<0.001\right)$ and a significant effect of test block $\left(\mathrm{F}_{13,161}=9.78, \mathrm{P}<0.001\right)$. These effects persisted when sex was included in the analysis. Likewise, on the $3 \mathrm{~min}$ PVT there was a significant effect of group $\left(\mathrm{F}_{1162}=17.40, \mathrm{P}<0.001\right)$ and a significant effect of test block $\left(\mathrm{F}_{13,162}=8.67, \mathrm{P}<0.001\right)$. When sex was included in the analysis, the effect of group was no longer statistically significant, while the effect of test block persisted. There was a significant effect of sex on mean RT in the 3 min PVT $\left(\mathrm{F}_{1134}=15.05, \mathrm{P}<0.001\right)$, with females exhibiting slower mean RT by $30 \mathrm{~ms}$ (SE, $8 \mathrm{~ms}$ ).

In both groups and for both versions of the PVT, mean RT was relatively fast during the first $16 \mathrm{~h}$ of wakefulness and then increased considerably across the $38 \mathrm{~h}$ TSD period, with peak impairment during the early to late morning and a partial rebound during the subsequent afternoon similar to what was observed for lapses. Likewise, following recovery sleep, mean RT values returned to the level observed during the TSD period. Again, for both versions of the PVT, the level of impairment seen during sleep deprivation was greater in those with sleep-onset insomnia than in the healthy normal sleepers (Figure 2, middle).

For number of false starts on the 10 min PVT, there was a significant effect of group $\left(\mathrm{F}_{1161}=36.55, \mathrm{P}<0.001\right)$ and a significant effect of test block $\left(\mathrm{F}_{13,161}=4.25, \mathrm{P}<\right.$ 0.001 ), as well as a significant group by test block interaction $\left(\mathrm{F}_{13,161}=2.14, \mathrm{P}=0.014\right)$. These effects persisted when sex was included in the analysis. For number of false starts on the 3 min PVT, there was a significant effect of test block $\left(\mathrm{F}_{13,162}=3.29, \mathrm{P}<0.001\right)$. When sex was included in the analysis, the effect of test block persisted,

Table 2 F Statistics for Analyses of PVT Outcome Measures

\begin{tabular}{|c|c|c|c|c|c|c|}
\hline & \multicolumn{2}{|l|}{ Lapses } & \multicolumn{2}{|l|}{ Mean RT } & \multicolumn{2}{|l|}{ False Starts } \\
\hline & $10 \mathrm{~min}$ PVT & $3 \mathrm{~min}$ PVT & $10 \mathrm{~min}$ PVT & $3 \mathrm{~min}$ PVT & $10 \mathrm{~min}$ PVT & $3 \mathrm{~min}$ PVT \\
\hline Main effect, group & $22.42 * * *$ & $22.45 * * *$ & $13.66 * * *$ & $17.40 * * *$ & $36.55^{* * * *}$ & 1.16 \\
\hline Main effect, test block & $11.95 * * *$ & $5.06 * * *$ & $9.78^{* * *}$ & $8.67^{* * *}$ & $4.25^{* * *}$ & $3.29 * * *$ \\
\hline Interaction, group by test block & 0.96 & 1.36 & 1.48 & 1.07 & $2.14^{*}$ & 0.44 \\
\hline Main effect, group & $5.57^{*}$ & 2.78 & $5.8 I^{*}$ & 1.60 & $13.27 * * *$ & $14.02 * * *$ \\
\hline Main effect, test block & $7.35 * * *$ & $1.88^{*}$ & $7.26 * * *$ & $4.07 * * *$ & $3.51 * * *$ & $3.38 * * *$ \\
\hline Interaction, group by test block & 0.80 & 0.35 & 1.67 & 0.35 & $\mathrm{I} .87^{*}$ & 0.47 \\
\hline Main effect, sex & 2.93 & $7.38 * *$ & 1.35 & $15.05 * * *$ & 0.01 & $15.38 * * *$ \\
\hline Interaction, group by sex & 0.17 & 1.53 & 0.26 & 0.19 & 1.93 & $8.11 * *$ \\
\hline Interaction, bout by sex & 0.36 & 0.87 & 0.44 & 1.18 & 1.05 & 0.56 \\
\hline Interaction, group by test block by sex & 0.34 & 0.23 & 0.76 & 0.39 & 0.61 & 0.91 \\
\hline
\end{tabular}

Notes: Top section, primary analysis; bottom section, secondary analysis controlling for sex. $* \mathrm{P}<0.05, * * \mathrm{P}<0.0 \mathrm{I}$, $* * * \mathrm{P}<0.00 \mathrm{I}$. PVT $=$ psychomotor vigilance test; RT = response time. 
Time Awake (h)

$\begin{array}{lllllllllllll}0 & 3 & 6 & 9 & 12 & 15 & 18 & 21 & 24 & 27 & 30 & 33 & 36\end{array}$
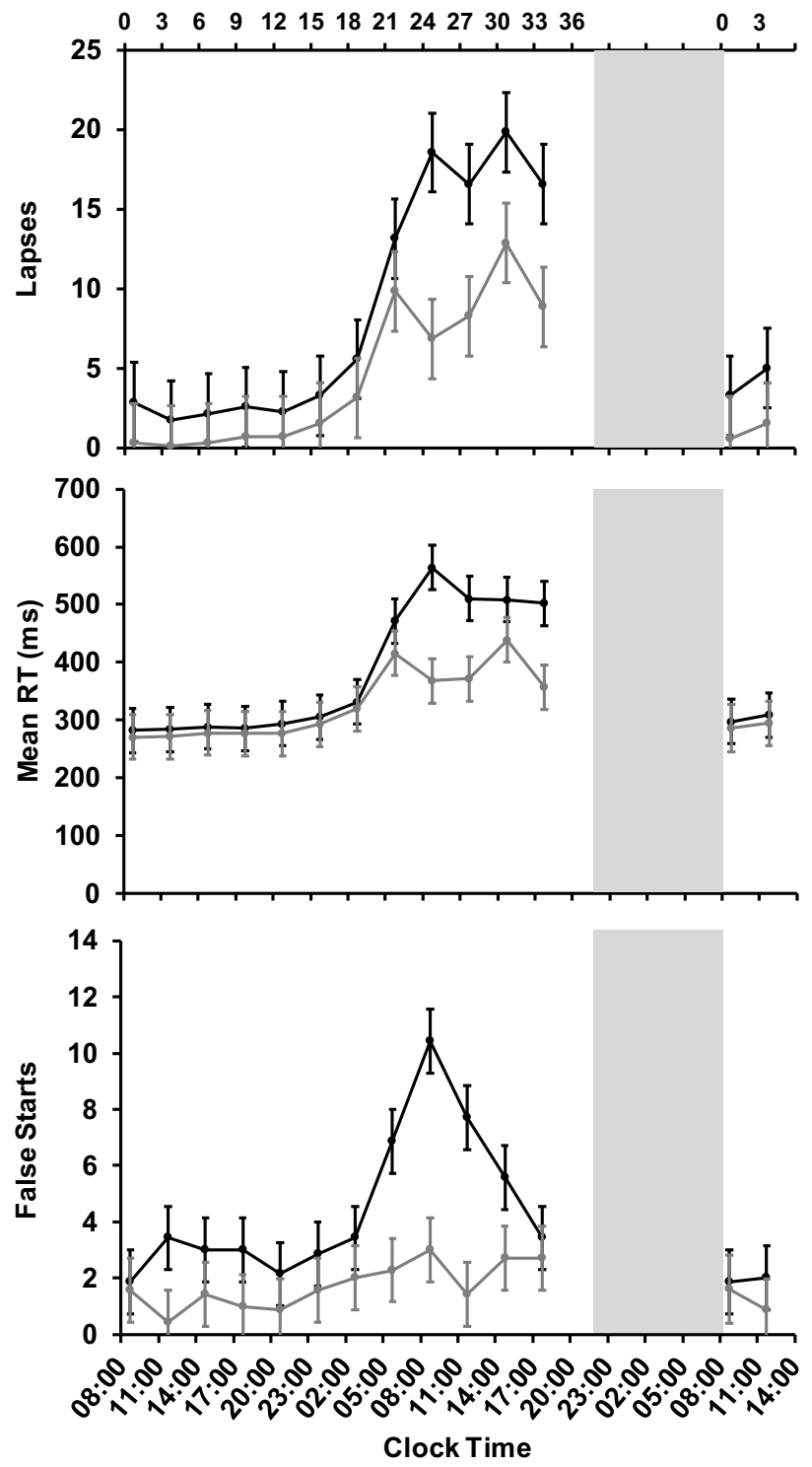

Time Awake (h)
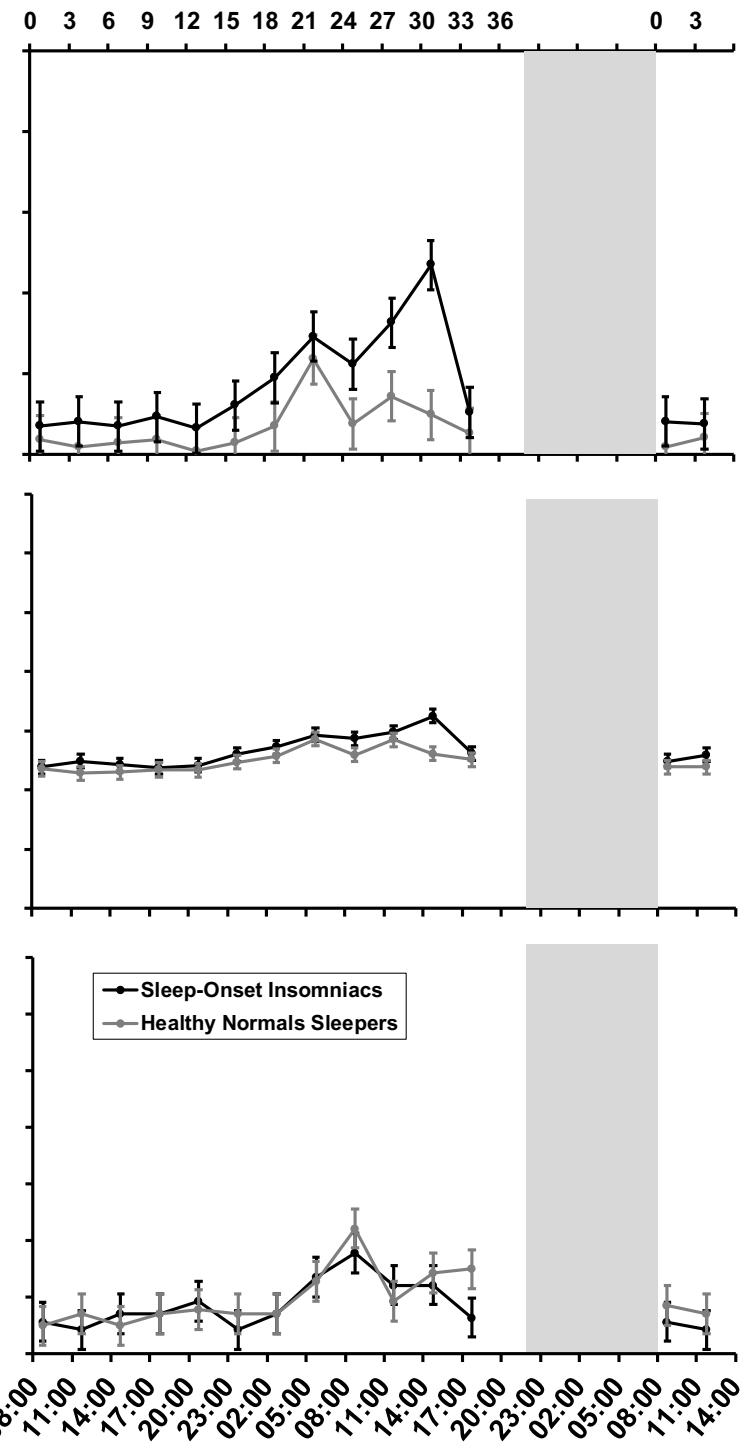

Clock Time

Figure 2 Psychomotor vigilance test results.

Notes: Means \pm SE for lapses of attention (top), mean RT (middle), and false starts (bottom) on the 10 min PVT (left) and the 3 min PVT (right) across $38 \mathrm{~h}$ of total sleep deprivation and following recovery sleep. Data are plotted against the start times of performance test blocks. Cumulative time awake is shown across the top. Black: sleeponset insomniacs; gray: healthy normal sleepers. Gray vertical bars: recovery sleep.

Abbreviations: PVT, psychomotor vigilance test; RT, reaction time.

and a significant effect of group emerged $\left(\mathrm{F}_{1134}=14.02\right.$, $\mathrm{P}<0.001)$. There was also a significant effect of sex on number of false starts in the 3 min PVT $\left(\mathrm{F}_{1134}=15.38\right.$, $\mathrm{P}<0.001$ ), as well as a significant group by sex interaction $\left(F_{1134}=8.11, P=0.005\right)$. In both groups, females exhibited more false starts on the 3 min PVT than males; across groups, the difference was 2.1 (SE, 0.5).

In accordance with the instruction to participants to avoid false starts, false starts were relatively rare throughout the experiment (Figure 2, bottom) - except on the 10 min PVT for the sleep-onset insomnia group, where the incidence of false starts was higher across the $38 \mathrm{~h}$ TSD period, especially during the early to late morning after extended wakefulness, in the sleep-onset insomnia group.

Figure 3 shows the results of the analyses of the time-on-task effect in the raw RT data divided in 1 min bins. For the $10 \mathrm{~min}$ PVT, there were significant effects of group, test block, bin, and their two- and three-way interactions (all $\mathrm{P}<0.001$ ). For the $3 \mathrm{~min}$ PVT, there were significant effects of group, test block, bin, group by test block interaction, and test block by bin interaction (all $\mathrm{P}<0.001$ ). 
These results demonstrate that the time-on-task effect (i.e., the rate of change in RT within a test block) was increased in conjunction with the overall level of impairment on the PVT. Similarly, following recovery sleep, the time-on-task effect was reduced to what was observed during the first $16 \mathrm{hrs}$ of wakefulness. As expected, the time-on -task effect was overall greater in the 10 min PVT than in the $3 \mathrm{~min}$ PVT due to the difference in task duration. On both the 10 min and 3 min PVTs, in proportion to the task duration, the sleep-onset insomnia group (as compared to the healthy normal sleeper group) showed greater time-ontask effects during sleep deprivation (Figure 3).

\section{Discussion}

In this highly controlled in-laboratory study, both the individuals with sleep-onset insomnia and age-matched normal sleepers produced low RTs on the PVT and exhibited few lapses of attention and few false starts during the regular day (first $16 \mathrm{~h}$ of the TSD period). Further, PVT performance degraded significantly during $38 \mathrm{~h}$ TSD in both groups, with mean RT, lapses, false starts, and the time-on-task effect showing the stereotypical interplay of the effects of time awake and time of day (Figures 2 and 3). That is, performance during sleep deprivation showed the usual homeostatic and circadian influences on the temporal profile of neurobehavioral functioning ${ }^{28,29}$ in both groups. However, the magnitude of PVT performance deficits during $38 \mathrm{~h}$ TSD was significantly exacerbated in the participants with chronic sleep-onset insomnia.

The greater magnitude of psychomotor vigilance performance impairment in the sleep-onset insomnia group was observed regardless of whether the PVT was $10 \mathrm{~min}$ or only $3 \mathrm{~min}$ in duration, indicating that non-specific short attention span in those with sleep-onset insomnia is not
Time Awake (h)

$0 \begin{array}{llllll}0 & 6 & 9 & 121518212427303336\end{array}$
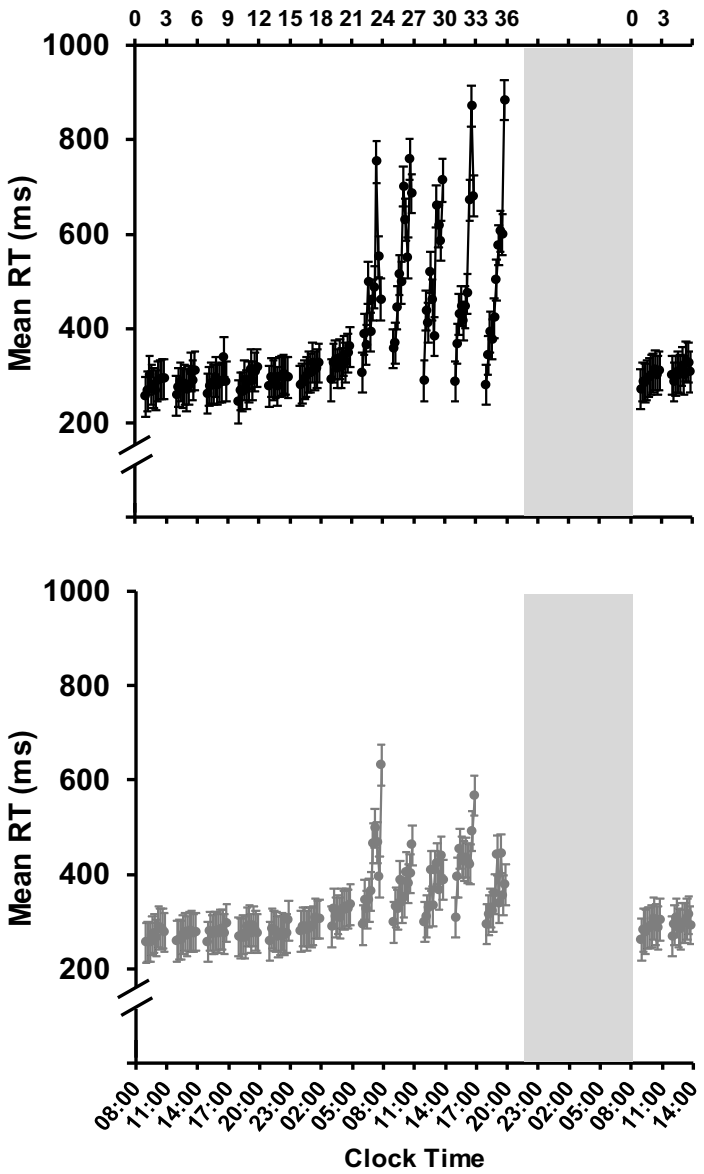

Time Awake (h)

$0 \quad 3 \quad 6 \quad 9121518212427303336$
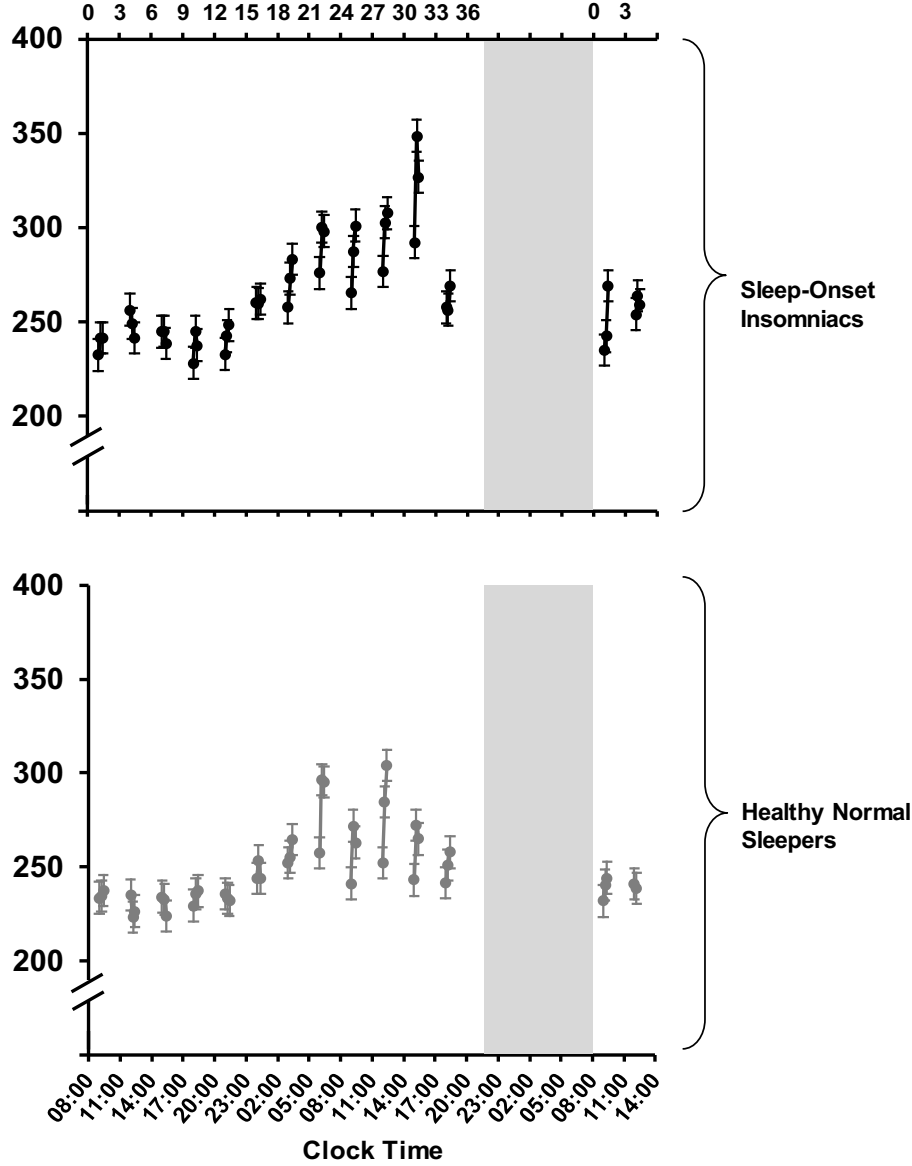

Figure 3 Time-on-task results.

Notes: Means \pm SE for RT in I min bins on the 10 min PVT (left) and the 3 min PVT (right) across $38 \mathrm{~h}$ of total sleep deprivation and following recovery sleep. Data are plotted against the start times of the test blocks; placement of the I min bins in each test block is not to scale on the clock time axis. Cumulative time awake is shown across the top. Note the difference in vertical scale between the graphs on the left (10 min PVT) versus right (3 min PVT). Black (top graphs): sleep-onset insomniacs; gray (bottom graphs): healthy normal sleepers. Gray vertical bars: recovery sleep.

Abbreviations: PVT, psychomotor vigilance test; RT, reaction time. 
a plausible explanation for the observed performance deficits. The exacerbated impairment during TSD in the insomnia group, as compared to the age-matched control group, cannot be attributed to differences in participant characteristics or baseline sleep beyond those directly related to sleep-onset insomnia (Table 1). Rather, our findings are consistent with previously hypothesized latent performance deficits in chronic insomnia, ${ }^{14}$ which patients may be able to compensate effectively during the normal day but are manifested during TSD.

Latent performance deficits that are not evident under baseline conditions but exposed by sleep deprivation have also been observed in healthy individuals previously sleep-restricted for multiple days. Evidence in adolescents and adults has indicated that although such individuals appear to recuperate to near-optimal performance levels following recovery sleep, they show heightened vulnerability to performance impairment when reexposed to sleep deprivation. ${ }^{30,31}$ Mathematical modeling results point to the involvement of allostatic changes in sleep homeostasis in response to sustained sleep restriction. ${ }^{32}$ This suggests that chronic sleep deficiency may cause a shift in the set point for sleep homeostasis, such that moderately sleep-deficient individuals are able to maintain near-optimal performance during baseline, but at the price of experiencing profound deficits when provoked by further sleep loss. ${ }^{33}$ It is possible that sleep deficiency in individuals with chronic insomnia induces the same phenomenon, and our results are consistent with this idea. A comparison between persons with chronic sleep-onset insomnia and healthy normal sleepers under laboratory-controlled conditions of sustained sleep restriction could help to elucidate this.

Our results are also consistent with the most widely posited mechanism underlying insomnia, namely hyperarousal. That is, it is possible that hyperarousal may mask latent daytime performance impairment, which is exposed when the sleep/wake system is further perturbed by TSD. ${ }^{34}$ If so, our results imply that hyperarousal does not provide any substantive protection against performance deficits when wakefulness is extended beyond the normal day, as manifest impairment during TSD was much greater in the sleep-onset insomnia group than in the agematched normal sleepers. Thus, as has been proposed before, any hyperarousal in sleep-onset insomnia may be bedtime-specific. $^{34}$

The individuals with chronic insomnia in our study were selected specifically for sleep-onset insomnia. Our results may thus be limited to this subpopulation of primary insomnia. Distinguishing sleep-onset insomnia from other insomnia subtypes is important, as the etiology and symptomology may be different, and this may explain the hitherto mixed results in the literature on performance impairment due to insomnia. Further research in individuals with sleep-maintenance insomnia may shed light on this issue.

The sample size in this study was relatively small, but we had considerable experimental control and statistical power due to strict laboratory control over measurement conditions, inclusion of an adaptation day and night to rule out any confounds from a (reverse) first-night effect, ${ }^{35}$ and the availability of an age-matched control group of healthy normal sleepers. In line with the higher prevalence of insomnia in females compared to males in the general population, ${ }^{36}$ however, females were overrepresented in the insomnia group as compared to the control group. Although we controlled for sex in our statistical analyses, a priori the difference between the groups in the magnitude of PVT performance impairment during sleep deprivation could be sex-related rather than insomnia-related. Yet, there is no evidence that sex per se systematically influences the effect of sleep deprivation on performance, ${ }^{37}$ so this alternative explanation of our results is unlikely.

\section{Conclusion}

The increased vulnerability to sleep deprivation in our sample of participants with chronic insomnia suggests that sleep-onset insomnia interacts fundamentally with sleep/wake regulation. Although individuals with sleeponset insomnia appear to be able to compensate effectively for psychomotor vigilance performance impairment during the normal day, ${ }^{38}$ they may be at increased risk of neurobehavioral deficits in settings that involve extended wakefulness. This is important, for example, with regard to workplace performance and the safety of persons with insomnia engaged in shift work and extended operations. In this context, it is also worth noting that the temporal dynamics (as opposed to the magnitude) of performance impairment over time awake, time of day, and time-ontask appear to be comparable to those of healthy normal sleepers (see Figures 2 and 3). Mathematical models of fatigue, which predict homeostatic and circadian influences on performance, ${ }^{39}$ may thus be useful for managing workplace performance impairment due to sleep loss in individuals with sleep-onset insomnia and healthy normal sleepers alike. 
In conclusion, our findings indicate that the complaints of persons with sleep-onset insomnia have both subjective and objective components. This underscores the importance of treatment for individuals with insomnia, so as to protect them from performance impairment and potential harm. Furthermore, our findings suggest that the effectiveness of different insomnia treatment options could be tested in laboratory sleep deprivation studies and evaluated objectively through the assessment of psychomotor vigilance performance.

\section{Acknowledgments}

We thank the study volunteers for their participation, and the staff of the Human Sleep and Cognition Laboratory in the Sleep and Performance Research Center at Washington State University for their assistance in data collection. This study was supported by Office of Naval Research grant N00014-13-C-0063 through Pulsar Informatics, Inc. The smartphone-based PVTs used in the study were on loan from Pulsar Informatics, Inc. This company was not involved in study design and execution, data analyses, and manuscript writing.

\section{Disclosure}

The authors report no conflicts of interest in this work.

\section{References}

1. Kessler RC, Berglund PA, Coulouvrat $\mathrm{C}$, et al. Insomnia and the performance of US workers: results from the America Insomnia Survey. Sleep. 2011;34(9):1161-1171. doi:10.5665/SLEEP.1230

2. American Academy of Sleep Medicine. International Classification of Sleep Disorders - Third Edition (ICSD-3). Westchester, IL: American Academy of Sleep Medicine; 2014.

3. Bonnet MH, Rosa RR. Sleep and performance in young adults and older insomniacs and normals during acute sleep loss and recovery. Biol Psychol. 1987;25(2):153-172. doi:10.1016/0301-0511(87)900 35-4

4. Bastien $\mathrm{CH}$. Insomnia: neurophysiological and neuropsychological approaches. Neuropsychol Rev. 2011;21(1):22-40. doi:10.1007/ s11065-011-9160-3

5. Fortier-Brochu E, Beaulieu-Bonneau S, Ivers H, Morin CM. Insomnia and daytime cognitive performance: a meta-analysis. Sleep Med Rev. 2012;16(1):83-94. doi:10.1016/j.smrv.2011.03.008

6. Shekleton JA, Flynn-Evans EE, Miller B, et al. Neurobehavioral performance impairment in insomnia: relationships with self-reported sleep and daytime functioning. Sleep. 2014;37(1):107-116. doi:10.5665/sleep.3318

7. Altena E, Van der Werf YD, Strijers RLM, Van Someren EJW. Sleep loss affects vigilance: effects of chronic insomnia and sleep therapy. J Sleep Res. 2008;17(3):335-343. doi:10.1111/j.1365-2869.2008.00671.x

8. Shekleton JA, Rogers NL, Rajaratnam SM. Searching for the daytime impairments of primary insomnia. Sleep Med Rev. 2010;14(1):47-60. doi:10.1016/j.smrv.2009.06.001

9. Liu H, Wang D, Li Y, et al. Examination of daytime sleepiness and cognitive performance testing in patients with primary insomnia. PLoS One. 2014;9(6):e100965. doi:10.1371/journal.pone.0100965
10. Kyle SD, Miller CB, Rogers Z, Siriwardena AN, MacMahon KM, Espie CA. Sleep restriction therapy for insomnia is associated with reduced objective total sleep time, increased daytime somnolence, and objectively impaired vigilance: implication for the clinical management of insomnia disorder. Sleep. 2014;37(2):229-237. doi:10.5665/sleep.3386

11. Belenky G, Wesensten NJ, Thorne DR, et al. Patterns of performance degradation and restoration during sleep restriction and subsequent recovery: a sleep dose-response study. J Sleep Res. 2003;12:1-12. doi:10.1046/j.1365-2869.2003.00337.x

12. Van Dongen HPA, Maislin G, Mullington JM, Dinges DF. The cumulative cost of additional wakefulness: dose-response effects on neurobehavioral functions and sleep physiology from chronic sleep restriction and total sleep deprivation. Sleep. 2003;2:117-126. doi:10.1093/sleep/26.2.117

13. Pillai V, Roth R, Drake CL. The nature of stable insomnia subtypes. Sleep. 2015;38(1):127-138. doi:10.5665/sleep.4338

14. Varkevisser M, Van Dongen HPA, Kerkhof GA. Physiological indexes in chronic insomnia during a constant routine: evidence for general hyperarousal? Sleep. 2005;28(12):1588-1596. doi:10.1093/ sleep/28.12.1588

15. Grant DA, Honn K, Layton ME, Van Dongen HPA. 3-minute smartphone-based and tablet-based psychomotor vigilance tests for the assessment of reduced alertness due to sleep deprivation. Behav Res Methods. 2017;49(3):1020-1029. doi:10.3758/s13428-016-0763-8

16. Johns MW. A new method for measuring daytime sleepiness: the epworth sleepiness scale. Sleep. 1991;14(6):540-545. doi:10.1093/ sleep/14.6.540

17. Buysse DJ, Reynolds CF, Monk TH, Berman SR, Kupfer DJ. The pittsburg sleep quality index: a new instrument for psychiatric practice and research. Psychiatry Res. 1988;28:193-213. doi:10.1016/ 0165-1781(89)90047-4

18. Smith CS, Reilly C, Midkiff K. Evaluation of three circadian rhythm questionnaires with suggestions for an improved measure of morningness. J Appl Psychol. 1989;74(5):728-738. doi:10.1037/00 21-9010.74.5.728

19. Bastien $\mathrm{CH}$, Vallieres A, Morin CM. Validation of the insomnia severity index as an outcome measure for insomnia research. Sleep Med. 2001;2(4):297-307. doi:10.1016/S1389-9457(00)00065-4

20. Newell J, Mairesse O, Verbanck P, Neu D. Is a one-night stay in the lab really enough to conclude? First-night effect and night-to-night variability in polysomnographic recordings among different clinical populations. Psychiatry Res. 2012;200(2-3):795-801. doi:10.1016/j. psychres.2012.07.045

21. Basner M, Mollicone D, Dinges DF. Validity and sensitivity of a brief psychomotor vigilance test (PVT-B) to total and partial sleep deprivation. Acta Astronaut. 2011;69(11-12):949-959. doi:10.1016/ j.actaastro.2011.07.015

22. Dinges DF, Powell JW. Microcomputer analyses of performance on a portable, simple visual RT task during sustained operations. Behav Res Methods Instrum Comput. 1985;17(6):652-655. doi:10.3758/BF03200977

23. American Academy of Sleep Medicine. The AASM Manual for the Scoring of Sleep and Associated Events: Rules, Terminology and Technical Specifications. Westchester, IL: American Academy of Sleep Medicine; 2018.

24. Lim J, Dinges DF. Sleep deprivation and vigilant attention. Ann N Y Acad Sci. 2008;1129(1):305-322. doi:10.1196/annals.1417.002

25. Van Dongen HPA, Belenky G, Krueger JM. Investigating the temporal dynamics and underlying mechanisms of cognitive fatigue. In: Ackerman PL, editor. Cognitive Fatigue: Multidisciplinary Perspectives on Current Research and Future Applications. Washington, DC: American Psychological Association; 2011:127-147. doi:10.1037/12343-000

26. Dorrian J, Rogers NL, Dinges DF. Psychomotor vigilance performance: neurocognitive assay sensitive to sleep loss. In: Kushida CA, editor. Sleep Deprivation: Clinical Issues, Pharmacology, and Sleep Loss Effects. New York, NY: Marcel Dekker; 2005:39-70. doi:10.3109/9780203998007 
27. Van Dongen HPA, Maislin G, Dinges DF. Dealing with inter-individual differences in the temporal dynamics of fatigue and performance: importance and techniques. Aviat Space Environ Med. 2004a;75(suppl 3)):A147-A154.

28. Van Dongen HPA, Dinges DF. Sleep, circadian rhythms, and psychomotor vigilance. Clin Sports Med. 2005;24(2):237-249. doi:10.1016/ j.csm.2004.12.007

29. Daan S, Beersma DG, Borbély AA. Timing of human sleep: recovery process gated by a circadian pacemaker. Am J Physiol Regul Integr Comp Physiol. 1984;246(2):R161-R183. doi:10.1152/ajpregu.1984. 246.2.R161

30. Banks S, Van Dongen HPA, Dinges DF. Neurobehavioral response to sleep restriction is influenced by pre-existing sleep debt. Sleep Biol Rhythms. 2007;5(suppl 1):A103. doi:10.1111/j.1479-8425.2007.002 93. $\mathrm{x}$

31. Lo JC, Lee SM, Teo LM, Lim J, Gooley JJ, Chee MW. Neurobehavioral impact of successive cycles of sleep restriction with and without naps in adolescents. Sleep. 2016;40(2):zsw042. doi:10.1093/sleep/zsw042

32. McCauley P, Kalachev LV, Smith AD, Belenky G, Dinges DF, Van Dongen HPA. A new mathematical model for the homeostatic effects of sleep loss on neurobehavioral performance. $J$ Theor Biol. 2009;256(2):227-239. doi:10.1016/j.jtbi.2008.09.012
33. Grant DA, Van Dongen HPA. Individual differences in sleep duration and responses to sleep loss. In: Shaw PJ, Tafti M, Thorpy MJ, editors. The Genetic Basis of Sleep and Sleep Disorders. Cambridge, UK: Cambridge University Press; 2013:189-196.

34. Varkevisser M, Kerkhof GA. Chronic insomnia and performance in a 24-h constant routine study. J Sleep Res. 2005;14(1):49-59. doi:10.1111/j.1365-2869.2004.00414.x

35. Hauri PJ, Olmstead EM. Reverse first night effect in insomnia. Sleep. 1989;12(2):97-105. doi:10.1093/sleep/12.2.97

36. Zhang B, Wing YK. Sex differences in insomnia: a meta-analysis. Sleep. 2006;29(1):85-93. doi:10.1093/sleep/29.1.85

37. Wesensten N, Belenky G, Kautz MA, Thorne DR, Reichardt RM, Balkin TJ. Maintaining alertness and performance during sleep deprivation: modafinil versus caffeine. Psychopharmacology (Berl). 2002;159(3):238-247. doi:10.1007/s002130100916

38. Varkevisser M, Van Dongen HPA, Van Amsterdam JGC, Kerkhof GA. Chronic insomnia and daytime functioning: an ambulatory assessment. Behav Sleep Med. 2007;5(4):279-296. doi:10.10 80/15402000701557425

39. Hursh SR, Balkin TJ, Van Dongen HPA. Sleep and performance prediction modeling. In: Kryger $\mathrm{MH}$, Roth T, Dement WC, editors. Principles and Practice of Sleep Medicine. 6th ed. Philadelphia, PA: Elsevier; 2016:689-696. doi:10.1016/B978-0-323-24288-2.00072-6
Nature and Science of Sleep

\section{Publish your work in this journal}

Nature and Science of Sleep is an international, peer-reviewed, open access journal covering all aspects of sleep science and sleep medicine, including the neurophysiology and functions of sleep, the genetics of sleep, sleep and society, biological rhythms, dreaming, sleep disorders and therapy, and strategies to optimize healthy sleep.
The manuscript management system is completely online and includes a very quick and fair peer-review system, which is all easy to use. Visit http://www.dovepress.com/testimonials.php to read real quotes from published authors. 